\title{
A New Defibrillation Mechanism: Termination of Reentrant Waves by Propagating Action Potentials Induced by Nearby Heterogeneities
}

\author{
Shuyue Han $^{1}$, Niels F Otani ${ }^{1}$, Valentin Krinski ${ }^{2}$, Stefan Luther ${ }^{2}$ \\ ${ }^{1}$ Rochester Institute of Technology, Rochester, NY USA \\ ${ }^{2}$ Max Planck Institute for Dynamics and Self-Organization, Gottingen, Germany
}

\begin{abstract}
Introduction. Recently, there has been a major effort to develop new, low-energy defibrillation methods that would be less damaging and less traumatic for the patient, and would save battery energy. However, these methods have not been entirely successful, due in part to an incomplete understanding of all the mechanisms present that may help or hinder the process of terminating the rotating waves present during fibrillation. Here we describe new mechanisms whereby a far-field electric field pulse terminates unpinned waves that are rotating in the vicinity of a blood vessel, plaque deposit or other heterogeneity in the gap junction conductivity. Methods. We ran a series of twodimensional computer simulations of a spiral wave rotating in the vicinity of a non-conducting obstacle. Application of a low-energy electric field pulse caused a semicircular action potential wave to be launched from the heterogeneity which then interacted with the rotating wave. Results and Conclusions: We found, that, when this interaction is combined with, importantly, the presence of nearby non-conductive boundaries, termination of the rotating waves can occur via a number of new mechanisms, over a wide range of timings of the electric field pulse, and for a number of different initial locations of the rotating wave. The mechanisms only require the rotating wave to be nearby, but not necessarily pinned to the heterogeneity, and thus extends the effectiveness of the electric field pulses used in low-energy defibrillation. Consideration of these mechanisms together with those already discovered could result in the development of improved, low-energy defibrillation protocols.
\end{abstract}

\section{Introduction}

Recently, to avoid the adverse side effects from highenergy defibrillation shocks, the use of a series of lowercurrent, far-field electric field pulses has been advanced as a low-energy method for terminating life-threatening cardiac arrhythmias [1,2], The pulses cause heterogeneities in intercell electrical conductivity, such as blood vessels and plaques, to act as "virtual" electrodes, emitting propagating action potential waves within the tissue [3]. With proper design, these waves have the potential to interact with fibrillatory waves, unpinning them from obstacles around which they are rotating, and terminating them.

Previously, we and other researchers have introduced and compared different mechanisms based on different models that terminate rotating waves using far-field electric field pulses. Ripplinger et al. [4] demonstrated that, with proper timing, an electric field pulse can dislodge and terminate a wave rotating around a heterogeneity. In onedimensional ring models, electric field pulses have been shown to be effective in terminating rotating waves $[5,6]$. Hornung et al. have developed a phase-scanning technique that was effective in terminating multiple pinned vortices when their their phases and locations are unknown [7].

In this paper, we identify several mechanisms that help or hinder the process of terminating rotating waves in the presence of an obstacle, and determine what stimulus timings and wave locations are associated with each mechanism. These results should be useful in suggesting stimulus protocols that can increase the rate of defibrillation success. In particular, we show new mechanisms by which waves launched from obstacles in the presence of an electric field pulse can be effective in terminating freely (unpinned) rotating waves.

\section{Methods}

We used the dimensionless Barkley equations [8] to model the electrical activity of the heart. The model is often used as a qualitative model in pattern forming systems that are well described by the interaction between activator and inhibitor components. It is ideally suited for modeling action potentials in the heart, as it is capable of modeling the primary structures present during fibrillation (i.e., spiral waves in 2D and scroll waves in 3D), yet is simple enough to allow diagnosis of its behavior. 
Success: Rotating wave is terminated
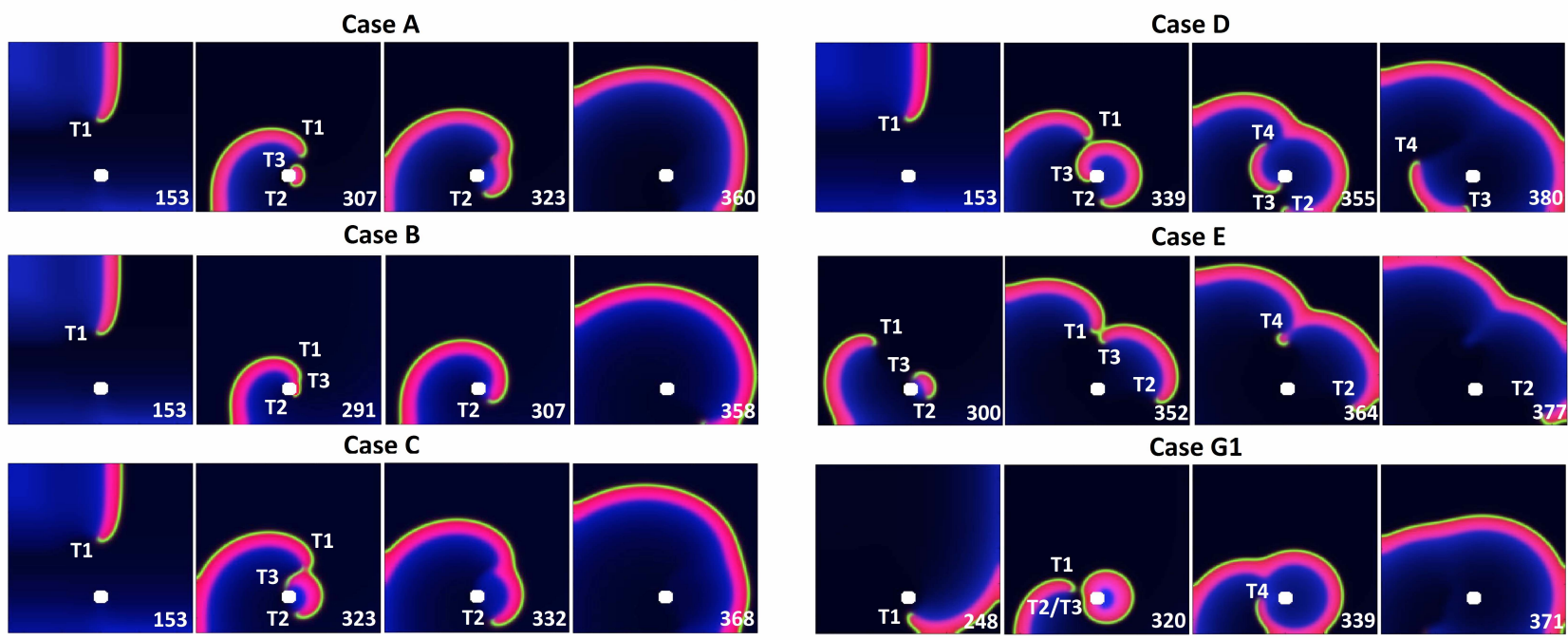

Failure: Rotating wave survives
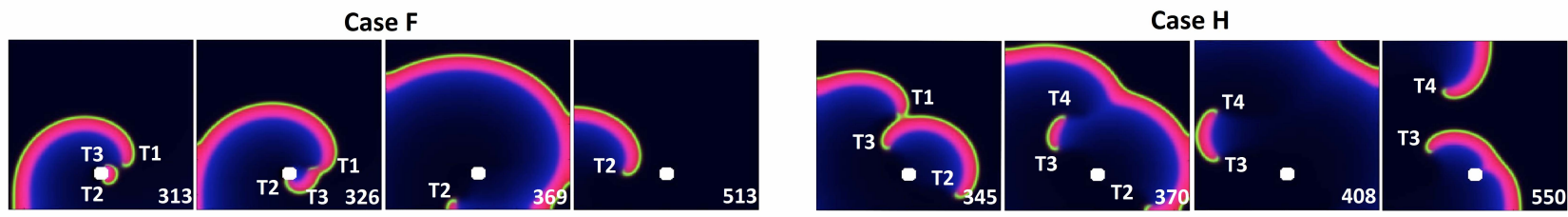

Case G2
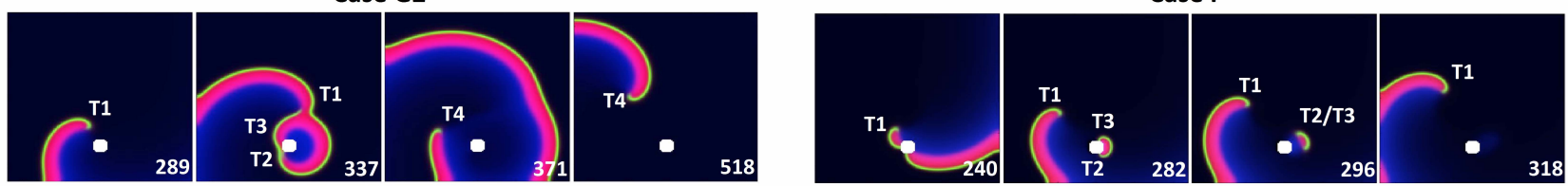

Figure 1. Ten cases showing the ten different types of behavior observed. Times appear in the lower-right hand corner of each snapshot. The rotating spiral wave is terminated by an E-pulse in the first 6 cases, but fails to be terminated in the last 4. The white disk represents the location of the obstacle. T1 is the tip of the free rotating spiral wave. T2 and T3 are the bottom and top tips, respectively, of the semicircular wave launched from the obstacle by the electric field pulse. T4 is the tip of a separated wave that sometimes appears when the rotating wave merges with the semi-circular wave. The green line represents the wavefront of the action potential. The red region defines the action potential, while the light blue region represents the refractory region.

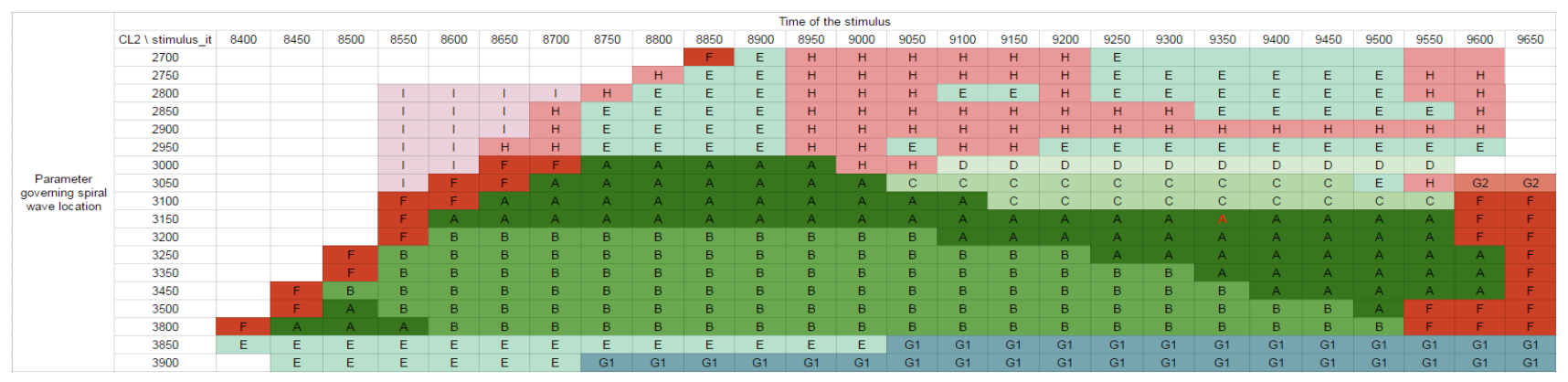

Figure 2. Table of outcomes from multiple runs from the computer simulation. The letter in each box corresponds to of the ten different cases. All greenish regions represent success cases, while all regions that are shades of red represent failure of the waves to terminate. 
The Barkley equations may be written as,

$$
\begin{gathered}
\frac{d u}{d t}=D_{u} \nabla^{2} u+\frac{1}{\epsilon} u(1-u)\left(u-\frac{v+b}{a}\right) \\
\frac{d v}{d t}=u-v,
\end{gathered}
$$

Here $u$ is the membrane potential (activator) and $v$ is the refractoriness of cells (inhibitor). The parameters $\epsilon$ is the ratio of their temporal scales. Parameters $a$ and $b$ characterize the activator kinetics. We defined $a=0.6$ and $b=0.075$, which puts the simulations in the regime of stably rotating spiral waves, rotating around a fairly large circular core. The parameter $\epsilon$ was set to be 0.02 . The computational grid spacing and timestep were chosen to be 0.1667 and 0.0016 , respectively. The membrane potential diffusion coefficient $D_{u}$ was set to be 1.0. Computer simulations were performed in a two-dimensional, rectangular system with no-flow boundary conditions on all system boundaries.

The purpose of our study was to see how we might be able to interfere with the rotating action potential waves that are the principal waves present during fibrillation. A representative, rotating spiral wave was therefore initiated in the system. Also, within the system, we placed a nonconducting, circular obstacle representing a heterogeneous feature of the tissue. At some point during each simulation, an electric field pulse (current $I=75$ in dimensionless units) was applied pointing horizontally, from right to left. We modeled the effect of this field pulse by applying the Neumann boundary conditions, $\hat{\mathbf{n}} \cdot \nabla u-\hat{\mathbf{n}} \cdot \mathbf{E}_{0}=0$, to the circular boundary of this obstacle [3]. Here $\hat{\mathbf{n}}$ is the local normal to the boundary, and $\mathbf{E}_{0}$ is the external electric field. Thus, during the electric field pulse, current effectively flowed out of the cells on the right side of the circular obstacle, causing depolarization of those cells, and then reentered the cells on the left edge of the obstacle, producing hyperpolarization [1]. The depolarization induced on the right side of the obstacle led to the formation of an action potential, which, in turn, generally initiated a new, propagating semicircular action potential wave. It is the interaction of this wave with the rotating wave that, in many cases, resulted in the termination of all wave activity. Note that the obstacle is located near the bottom border purposely, as it turns out that nearby boundaries play a role in the wave termination.

\section{Results}

In the Barkley model, it is known that propagating waves fail, when: (i) the refractory quantity is too large (specifically, when $v>a / 2-b$ ), (ii) the wavefront curvature becomes too large, or (iii), the wave runs into one of the (non-conducting) system boundaries. A subcase of (i) is the case of wavefronts running into each other, which results in mutual annihilation. We can use these properties to understand the 10 different types of behavior we observed in our simulations, as we varied (a) a parameter used in creating the rotating wave and (b) the timing of application of the electric field pulse:

Case A in Figure 1 shows the "default" mechanism for terminating a free spiral wave, to which we will compare the other cases. A free vortex with vortex tip T1 and the circular obstacle (in white) are shown at time $153 \mathbf{~ m s}$. At $307 \mathrm{~ms}$, a semicircular wave was formed by the electric field pulse (E-pulse) with vortex tips T2 and T3. At $\mathbf{3 2 3}$ ms, the spiral wave tip T1 merged with T3. When the spiral wave tip merges with another tip, there is no possibility of forming a new vortex. Then at $\mathbf{3 6 0} \mathbf{~ m s}$, the spiral wave tip T2 runs into the bottom border. Since the trailing edge of the spiral wave had only recently passed by the obstacle before the electric field was applied, the wavebreaks T2 (and also T3) could not propagate to the left because a residual refractory region (light blue) was still present there. Thus, T2 annihilated with the bottom wall. This tipto-tip merging case therefore resulted in the termination of the freely rotating spiral wave.

The other success cases shown in the Figure 1 have slightly different behaviors than Case $\mathbf{A}$, but the rotating wave is terminated in each case. In Case $\mathbf{B}$, the only difference is that the spiral wave was still pinned (i.e., attached) to the obstacle when the E-pulse was applied. Thereafter, $\mathrm{T} 1$ still merged with $\mathrm{T} 3$, and $\mathrm{T} 2$ ran into the bottom wall. In Case $\mathbf{C}$, instead of a tip-to-tip merge, T1 ran into the wavefront of the semicircular wave. In Case D, T1 broke the semicircular wave into two pieces. The smaller piece had two tips T3 and T4, which annihilated after running int the bottom wall and the left wall, respectively. In Case $\mathbf{E}$, the wavefronts of both rotating wave and semicircular wave merged with each other, creating a second, much smaller, separate wave. However, this wave died due to the high curvature of its wavefront.

In most of the cases in which termination failed, the wave tip came close to a boundary, but did not run into it. Case F corresponds to Case A, but T2 didn't annihilate with the bottom wall. Instead, T2 spiraled back into the medium. Similarly, Case $\mathbf{H}$ exhibited the same spiral wave behavior as Case D, except T3 and T4 had room to spiral back around into a pattern called figure-of- 8 reentry. The exception is Case I, where the depolarized region induced adjacent to the obstacle failed to propagate, and thus was unable to interact with the spiral wave.

This leaves Cases G1 and G2, which operated with a mechanism totally different from all the other cases. In these cases, the E-pulse was applied so early that the semicircular wave was able to propagate all the way around 
the obstacle, creating an expanding circular wave. In Case G1, the rotating spiral wave was terminated as it ran into a boundary, while the wave survived in Case G2.

These different cases were produced by varying the timing of when the electric field was applied, and varying the initial location of the spiral wave relative to the location of the obstacle and boundaries. The table in Figure 2 shows how these two parameters affected the outcome. We observe that the initial location of the spiral wave affected the outcome more than the timing of the stimulus. However, termination occurred over a fairly wide range of variation in both parameters.

\section{Discussion}

In all the success cases, the induced wave emitted from the obstacle worked together with the spiral wave tip of the rotating wave and the left and bottom boundaries to produce termination of all wave activity. Furthermore, in cases in which termination failed, the wave tip came close to a boundary, but did not run into it. Thus, these simulations show that, at least for the mechanisms studied and the simulation parameters used, the presence of boundaries is a key factor in terminating freely rotating spiral waves.

We observe that, for a given set of simulation parameters, this is really a three-parameter initial value problem. The outcome depends on (1) the distance the spiral wave tip is from the left boundary when the spiral wave arm is oriented in some reference direction, for example, upwards, (2) the distance the tip is from the bottom boundary at the same time, and (3) the period of time that elapses between this reference configuration of the spiral wave and the application of the electric field pulse. Our next goal will be search this 3D parameter space, and develop a 3D table analogous to the one shown in Fig 2.

Limitations. To date, we have only looked at one set of parameters $(a=0.6, b=0.075)$. In the future, we will examine other parameter regimes. In our parameter regime, the spiral wave stably rotates around a relatively large circular core, which allows it to come closer to the boundaries making it easier to terminate. This is representative of the real case, in the sense that reentrant waves in the heart tend to rotate around linear or Z-shaped cores of finite length, and so are also susceptible to termination by approaching and running into non-conducting structures in the heart. However, other parameter regimes should be examined.

\section{Conclusions}

We studied termination mechanisms for freely rotating spiral waves by delivering a low-energy far-field electric field pulse that interacts with rotating waves through its induction of propagating, semicircular waves from nearby heterogeneities. For the parameters we studied, the ini- tial location of the spiral wave relative to the boundaries and the obstacle was a key factor in the success or failure of terminating freely rotating spiral waves. We found that several related wave termination mechanisms were collectively able to produce termination for a variety of stimulus times and spiral wave locations relative to an obstacle and the boundaries. We initially conducted these simulations to study the possibility that tip-to-tip interactions can result in termination of the spiral wave. However, we have found that tip-to-wavefront and the interaction with the tip with residual partially refractory regions and the boundaries were also effective in producing wave termination.

\section{References}

[1] Fenton FH, Luther S, Cherry EM, Otani NF, Krinsky V, Pumir A, Bodenschatz Jr. RF. Termination of atrial fibrillation using pulsed low-energy far-field stimulation. Circulation 2009;120:467 - 476.

[2] Luther S, Fenton FH, Kornreich BG, Squires A, Bittihn P, Hornung D, Zabel M, Flanders J, Gladuli A, Campoy L, Cherry EM, Luther G, Hasenfuss G, Krinsky VI, Pumir A, Gilmour Jr. RF, Bodenschatz E. Low-energy control of electrical turbulence in the heart. Nature 2011;475:235-239.

[3] Pumir A, Krinsky V. Unpinning of a rotating wave in cardiac muscle by an electric field. $J$ Theor Biol 1999;199:311-319.

[4] Ripplinger CM, Krinsky VI, Nikolski VP, Efimov IR, Mechanisms of unpinning and termination of ventricular tachycardia. Amer. J. Physiol. Heart Circulat. Physiol. 2006;291: H184.

[5] Otani NF. Termination of reentrant cardiac action potential ropagation using far-field electrical pacing. IEEE Transactions on Biomedical Engineering 2011;58(7):2013-2022.

[6] Bragard J, Simic A, Elorza J, Grigoriev RO, Cherry EM, Gilmour Jr. RF, Otani NF, Fenton FH. Shock-induced termination of reentrant cardiac arrhythmias: comparing monophasic and biphasic shock protocols. Chaos 2013;23(4):043119.

[7] Hornung D, Biktashev V, Otani NF, Shajahan TK, Baig T, Berg S, Han M, Parlitz U, Krinsky V, Luther S. Mechanisms of vortices termination in the cardiac muscle. Submitted.

[8] Barkley D.Euclidean symmetry and the dynamics of rotating spiral waves. Phys. Rev. Lett. 1994;72:164.

Address for correspondence:

Shuyue Miki Han

School of Mathematical Sciences

Rochester Institute of Technology

Rochester, NY 14624 USA

sxh8216@rit.edu 